Canad. Math. Bull. Vol. 19 (3), 1976

\title{
A NOTE ON ABSOLUTELY PURE MODULES
}

\author{
BY \\ EDGAR ENOCHS
}

Fieldhouse observed that any finitely presented left $R$-module $P$ is projective with respect to pure exact sequences, i.e.

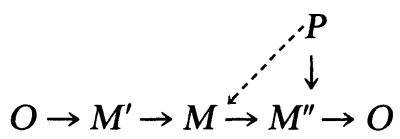

can always be completed to a commutative diagram when the sequence is pure exact. A left $R$-module $A$ is absolutely pure if it is a pure submodule of every module which contains it. Hence $\operatorname{Ext}_{R}^{1}(P, A)=0$ if $P$ is finitely presented and $A$ is absolutely pure.

The object of this note is to show that finitely presented modules $P$ are characterized among the finitely generated modules by the property that $\operatorname{Ext}_{R}^{1}(P, A)=0$ for all absolutely pure modules $A$. If $0 \rightarrow S \rightarrow F \rightarrow P \rightarrow 0$ is exact where $F$ is free on a finite number of generators then $\operatorname{Ext}_{R}^{1}(P, A)=0$ if and only if

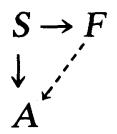

can always be completed to a commutative diagram. $P$ is finitely presented if and only if $S$ is finitely generated. Hence it suffices to prove that if the diagram can always be completed to a commutative diagram then $S$ must be finitely generated. This turns out to be true even if we drop the hypothesis that $F$ be free and only require that it be finitely generated.

Proposition. If $S \subset M$ is a submodule of a finitely generated module $M$, then if for every absolutely pure module $A$ and any linear map $S \rightarrow A$<smiles>[Y14][Y14]1=CS1</smiles>

can be completed to a commutative diagram, $S$ is finitely generated.

Proof. Suppose $S$ is not finitely generated. Let $X \subset S$ be a set of generators of least possible cardinality. Well order $X$ so that $X$ has no largest element and so that every section has cardinality smaller than the cardinality of $X$. For each 
$x \in X$ consider the module. $\sum_{y \leq x} R y / \sum_{y \leq x} R y$. Imbed this module in an injective module $I_{x}$. Extend the obvious linear map $\sum_{y \leq x} R y \rightarrow I_{x}$ to a linear map $S \rightarrow I_{x}$. Let $G \subset \prod I_{x}$ consist of all families $\left(i_{x}\right) x \in X$ such that for some $z \in X, i_{x}=0$ if $x \geq z$. Then $G$ is the union of a collection of direct summands of $\prod_{x \in X} I_{x}$ which is filtering to the right; and hence $G$ is a pure submodule. Since $\Pi I_{x}$ is injective, $G$ is absolutely pure. Also the image of $S$ is contained in $G$.

By hypothesis the linear map $S \rightarrow G$ can be extended to a linear map $M \leftarrow G$. $M$ is finitely generated and $G$ is the union of the submodules $\prod_{y \leq x} I_{y}$ for $x \in X$ so that the image of $M$ would be contained in one of the submodules. But if the image of $S$ were in $\prod_{y \leq x} I_{y}, S$ would be generated by the section of $X$ determined by $x$. This contradicts the choice of $X$ and the order imposed on it.

\section{REFERENCES}

1. B. H. Maddox, Absolutely Pure Modules, Proc. Amer. Math. Soc. 18 (1967) 155-158.

2. C. Megibben, Absolutely Pure Modules, Proc. Amer. Math. Soc. 26 (1970) 561-566.

UNIVERSITY OF KENTUCKY

LEXINGTON, KENTUCKY 40506

U.S.A. 